\title{
NOVA SKED: A behavioral notation language for Data General minicomputers
}

\author{
STEVEN G. GILBERT \\ Department of Radiation Biology and Biophysics \\ University of Rochester Medical School, Rochester, New York 14627 \\ and \\ DEBORAH C. RICE \\ Health Protection Branch, Food Directorate \\ Health and Welfare Canada, Tunney's Pasture, Ottawa, Ontario, Canada K1A OL2
}

\begin{abstract}
A state notation language (NOVA SKED) for the experimental control and collection of data from operant behavior experiments by Data General NOVA series minicomputers is described. NOVA SKED is based on the SKED system written for the Digital Equipment Corporation PDP8 series of minicomputers. The NOVA SKED state notation syntax enables diagramming experimental procedures directly and precisely by the operant experimenter, who need not be familiar with computers. This syntax can then be compiled by the computer into programs that operate under the SKED run-time system (RTS). The SKED RTS operates in a timesharing mode that allows up to 16 experimental stations to function simultaneously and independently from each other. NOVA SKED is a "stand-alone" system that collects and stores data on digital magnetic tape.
\end{abstract}

NOVA SKED ${ }^{1}$ is a language designed for the experimental control and collection of data from operant behavior experiments using the Data General minicomputers. This system is compatible with the entire Data General line of computers, from the oldest NOVA through the Eclipse. The basic philosophy of the system, as well as the syntax of the language, has been adapted from the state notation language SKED written for the PDP-8 series of computers (Snapper, 1973; Snapper \& Kadden, 1973; Snapper, Stephens, Cobez, \& Van Haaren, Note 1), although the system differs in some details. There were several reasons for writing an "operant behavior language" for the NOVA: (1) The NOVA is a 16-bit machine, which is advantageous for two reasons. First, the efficient form of addressing in the instruction set allows the SKED run-time system to be written using reenterant code, thus enabling a program to be represented only once in memory even though it may be running more than one station simultaneously. Second, the 16-bit word allows a number as large as 65,535 to be stored before double precision must be used. This feature saves memory and generates considerable flexibility in the data collection format. (2) Most modern Data General machines are significantly faster than the PDP-8 series. (3) The Data General operating system RDOS is considerably more sophisticated than the PDP.8 operating system (OS-8), allowing more flexibility in file structure, for example. This advantage has been negated to an extent by revisions of OS-8 by Snapper and others in the new SUPER SKED (Snapper et al., Note 1).

\section{GENERAL DESCRIPTION}

SKED is a language designed for convenient use by the operant conditioning experimenter, who may or may not be familiar with computers. The syntax is based on the "state notation" concept (Snapper, Knapp, \& Kushner, 1970), a method for diagramming an experimental procedure directly and precisely that can be translated easily into the SKED syntax. This syntax is then compiled by the SKED compiler into a "state table," as the compiled version of the experimenterproduced programs is called, that is readable by the SKED run-time system (RTS). The RTS functions as a "stand-alone" system; that is, one that does not 'use the Data General operating system RDOS. This RTS has several features that facilitate the experimental analysis of behavior that are impossible or at least very difficult with other types of higher level languages (FORTRAN, COBAL, BASIC, etc.). These functions include the following:

(1) Each individual experiment can be programmed independently, without regard for the fact that different experiments may run simultaneously, or that the same program will be running in multiple stations. (2) Stations may be started and stopped completely independently, regardless of whether other experiments are running. Programs may also be loaded into memory (an operation 
distinct from starting a program in a particular station) via the paper-tape reader while experiments are running. (3) An initial dialogue with the RTS establishes the number of inputs and outputs for each box as well as translating the absolute address of each input or output (i.e., board number, bit number) into a relative address for that station that is used by the state table. In other words, "output 1" can represent the feeder in a program run in several stations, although the actual address for these outputs would differ for each station. This feature allows the same relocatable binary program (state table) to be run in different stations. This can be accomplished by a "bookkeeping" system external to the program itself.

Data collection in the SKED system is flexible in that data may be collected in detail ranging from the "relay rack counter" strategy to recording every interevent time down to within $10 \mathrm{msec}$. The latter is facilitated by means of two independent code systems, which the user can specify for coding different classes of events.

Hardware requirements include a Data General minicomputer with $32 \mathrm{~K}$ of memory, a disk, and the capability of running FORTRAN IV under RDOS control. Such a system is necessary for the SKED compiler, which is written in FORTRAN IV. In fact, the SKED compiler can be implemented on any 16-bit computer capable of running FORTRAN. The SKED RTS requires less than $2 \mathrm{~K}$ of memory and does not utilize the disk. This system supports magnetic tape as the data collection device, although data may be printed instead to a Teletype via a RTS keyboard command. The minimal requirements for the SKED RTS allow a small (4-8K) NOVA to be dedicated to the running of the experimental stations, with a larger system to be used for data analysis and compilation of SKED programs, although one machine may (of course) be used for all functions. If two machines are used, both the SKED RTS and the SKED relocatable binaries may be transferred between machines via mag tape or paper tape.

\section{SYNTAX}

Since this article is not intended as a manual, only a brief description of the SKED language syntax is given. The most important concept is that of "state notation," in which all specifications of the program may be represented graphically. The basic unit of the SKED syntax is the state. Each state represents a portion of the experimental procedure and may include changes of stimulus output. Control of movement from one state to the next is also specified in each state. For example, the basic contingencies of a DRL $20 \mathrm{sec}$ can be represented as shown in Figure 1.

In this example, an experimenter-produced event (R17) starts the procedure with the simultaneous

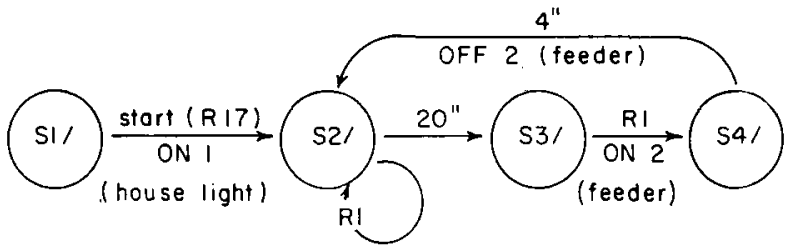

Figure 1. Basic contingencies of a DRL 20-sec schedule.

onset of Stimulus 1, in this case a houselight. Control is passed to State 2, or S2. When $20 \mathrm{sec}$ have elapsed, control is passed to S3, where a response on Lever 1 (R1) turns on Stimulus 2 (the feeder) and passes control to State 4. After $4 \mathrm{sec}$ have elapsed in $\$ 4$, the feeder is turned off and control is passed back to $S 2$ for another cycle. If the subject presses the lever (R1) in S2 before the $20 \mathrm{sec}$ have elapsed, however, the timer is reset and the interval begins again. This is represented by the arrow circling back to $\mathbf{S 2}$ from $\mathrm{S} 2$.

Each state consists of three parts: an input that triggers the change in state (in this example, time or a response by the subject), an output section, and a transfer of control to a specified state. The DRL $20 \mathrm{sec}$ may be coded in SKED language as follows, with comments denoted by a " $/$ ":

\section{S.S.1,}

S1,

R17:ON1 $\longrightarrow$ S2 /a response from the keyboard /by the experimenter tums on / the houselight and passes / control to S2.

S2,

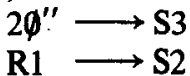

S3, $\mathrm{R} 1: \mathrm{ON} 2 \longrightarrow \mathrm{S} 4$ S4, $4^{\prime \prime}: \mathrm{OFF} 2 \longrightarrow \mathrm{S} 2$

This set of states controlling the contingencies of the DRL 20 sec comprise a subset of what is called a state set, designated S.S. (S.S.1 in the present example). Each state set runs independently but simultaneously with others in the program. One state in each state set is active at any time, with the program beginning at State 1. In the present example, no provision has been made for session timing, recording data such as IRT distribution, or operating a cumulative recorder. Some of these functions would be awkward to include in the existing state set but easily programmed in a separate state set. For example, a session timer for the DRL 20 sec could be coded in the following fashion: 
S.S.2,/ session timer

S1,

$\mathrm{R} 17 \longrightarrow \mathrm{S} 2 \quad /$ start timing under experi-

S2, /menter's control.

$6 \emptyset^{\prime} \longrightarrow$ STOP / session ends after $60 \mathrm{~min}$.

This state set would run independently of S.S.1 (control of the DRL) and stop the session after $60 \mathrm{~min}$.

The SKED syntax is divided into three parts: an input expression, an output expression, and a transition expression. The basic format is the following:

input(s): output(s) $\longrightarrow$ transition expression.

Inputs may be defined as the events that initiate processing of output functions and implement the transition to the next appropriate state. These inputs do not consist solely of inputs from the stations in the form of responses, but may also include time, as in the DRL 20-sec example, as well as other items discussed below. Similarly, outputs consist of any function initiated by a specified change in input conditions, and include more than just the turning on and off of stimuli in the experimental chambers.

NOVA SKED is sophisticated in terms of the kinds of functions it is able to perform. A brief list of functions provides some idea of the capabilities of the language.

\section{Inputs}

In addition to time and lever responses, an entity known as a $\mathrm{Z}$ pulse may be used to trigger state transitions. This is a "response" internal to the program which may be used to synchronize events between state sets. For example, if a $Z$ pulse (Z1) were generated every time a reinforcement occurred in the DRL 20-sec example, the session could also be ended contingent upon the number of reinforcements as well as by time:

$$
\begin{aligned}
& \text { S2, } \\
& 6 \emptyset^{\prime} \longrightarrow \text { STOP } \\
& 5 \emptyset \mathrm{Z1} \longrightarrow \text { STOP. }
\end{aligned}
$$

The session would end when the subject had received 50 reinforcements or when $60 \mathrm{~min}$ had elapsed, whichever happened first.

NOVA SKED allows the use of variables in the input expression, provided these have been previously set with an output expression. The following would be legal inputs:

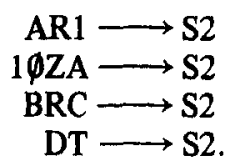

Other functions can be logically "anded" to the three basic input types (Rs, Zs, and time), so that transition from a state depends on the occurrence of two or more events. Inputs may be gated ("anded") to the value of a variable, the currently active state of a tagged state set, or a probability value. There may also be a default transition if none of the other requirements are met. An illustrative example is the following:

$$
\begin{aligned}
& 1 \emptyset \mathrm{R} 1 \& \mathrm{~A}(1,2) \& \mathrm{P}(5 \phi \emptyset) \longrightarrow \mathrm{S} 2 \\
& \text { \& } B(3) \& P(250) \longrightarrow S 3 \\
& : \longrightarrow \text { S4. }
\end{aligned}
$$

In this example, $A$ and $B$ can either be tagged state sets (e.g., S.S.1 = A) or they can be variables. Transition to S2 occurs with a probability of .500 if S.S.1 is in either State 1 or State 2 (or the variable $A$ is equal to either 1 or 2) when $10 \mathrm{R} 1 \mathrm{~s}$ have occurred. If these conditions are not met, then transition to S3 occurs with a probability of .250 if $10 \mathrm{R} 1 \mathrm{~s}$ have occurred when the state set tagged with B is in State 3 (or the variable $B$ is equal to 3 ). If this transition does not occur, then control passes to State 4.

\section{Outputs}

SKED outputs include turning stimuli on or off, Z-pulse production, data collection and output to mag tape, operations on variables (including incrementation and decrementation), choosing numbers from a list in a fixed sequence, and choosing from a list without replacement. In addition, the SKED system allows easy insertion into the body of a SKED program of user-generated machine language routines to perform special functions.

Variables may be either set or operated on in an output expression. A variable is set with the F2 function:

$$
\mathrm{Z1}: \mathrm{F} 2(\mathrm{~A}, 3) \longrightarrow \mathrm{S} 3 \text {. }
$$

In this example, a $Z 1$ sets $A$ equal to 3 . The variable $A$ may then be operated on by other functions, such as incrementation or decrementation by the $F 1$ function:

$$
\mathrm{R} 1: \mathrm{F} 1(\mathrm{~A},-1, \emptyset) \longrightarrow \mathrm{S} 1 \text {. }
$$

$A$ response on $R 1$ decrements the variable $A$ by one each time this line is executed, down to a minimum of zero. Once set, a variable may be used in input expressions, as discussed in the previous section; variables may also be utilized in output expressions. The following are legal expressions:

$$
\begin{aligned}
& \mathrm{R} 3: \mathrm{ONA} ; \mathrm{OFFB} \longrightarrow \mathrm{S} 2 \\
& \mathrm{R} 1: \mathrm{CG} \longrightarrow \mathrm{S} 2 \\
& 10^{\prime \prime}: \mathrm{ZC} \longrightarrow \mathrm{S} 1 .
\end{aligned}
$$

In the first expression, a response on $\mathrm{R} 3$ will turn on the stimulus corresponding to the value of $A$, and turn off the stimulus corresponding to $B$. In the second 
expression, an Rl will put a count in the counter corresponding to the current value of $G$. The third expression initiates a $Z$ pulse equal to the current value of $\mathrm{C}$ after $10 \mathrm{sec}$ have elapsed.

\section{Transitions}

Transition may be made to a different state within the state set, the same state (in which the state is "reset," as in the DRL example), or no change in state, in which any output operations for that line are performed but the status of the state is not changed. A transition may also be made to STOP, which inactivates all input and output lines to a particular station and thereby ends the session.

\section{DATA COLLECTION}

There are two basic strategies for data collection in SKED. One is the "relay rack" approach, in which each counter represents a certain event (e.g., Counter 1 may be the number of reinforcements, Counter 2 may be a postreinforcement-pause time clock, etc.). Data may be recorded at the end of the session, or the counter values may be written to the mag tape at specific intervals during the session, for example, every $10 \mathrm{~min}$. Each time the data is recorded on tape, all counters are automatically reset to zero. The other strategy is to record all interevent times as they occur. This typically would be done with double-precision counters, so that 32 bits are available to record both the interevent time itself (to any accuracy up to $10 \mathrm{msec}$ ) and a code identifying the event type. NOVA SKED contains a CODE function allowing the coding of 64 unique classes of events, which are assigned by the user. For example, Code 1 = fixed interval response, Code 2 = responses during reinforcement, and so on. This code occupies 6 bits of a double-precision word, leaving 26 bits for the interevent time itself, or 7.7 days using a 10-msec clock. A second code, called SUPER CODE, may also be added to a double-precision word, that can code 32 events. SUPER CODE occupies 5 bits, leaving 21 bits available to code the interevent time. The SUPER CODE function was developed for use in titration schedules, where it is convenient to code the intensity of the stimuli independent of the response type.

\section{RUN-TIME SYSTEM}

Minimum hardware requirements for the RTS are a NOVA, Teletype, and input and output hardware to service the experimental chambers. A mag tape is necessary if mass storage of data is required. Two versions of NOVA SKED have been designed to accommodate different types of interface hardware, either the Data General DIO boards or a system utilizing a separate hardware chassis and one slot in the main
NOVA chassis. ${ }^{2}$ With either system, the user must inform the RTS of the specific hardware organization. The device code and card numbers, if applicable, are passed to the RTS in an initial dialogue. The number of input and output bits is specified for each box, and each "box bit" is matched to the actual hardware bit number. Bits are assigned on a "one-to-one" basis with no restriction on which bits are associated with a particular station. In contrast, Snapper's SKED used a "mask" system that restricts bit assignment across device codes. There may be up to 64 of both input and output bits per station. Once this initial assignment has been made, the individual bit assignments for a particular station may be changed without changing the number of input and output bits associated with that station. This is handy for trouble-shooting hardware and allows a bit for a particular chamber to be reassigned without reassigning the whole system.

Compiled SKED programs are loaded by the RTS from either paper or magnetic tape. These relocatable binary state tables are available to the SKED system for loading into specific stations unless they are eliminated from memory with a SKED monitor command. A core image of the system may be written to mag tape at any phase of its generation. This is useful as a back-up for a small NOVA that will only be used for the SKED RTS, or as the storage mechanism for a SKED system that will be deleted and reloaded routinely. Thus a system that contains all bit assignments and all state tables currently being used may be saved to be reloaded with one operation. The number of programs that may reside in core at any time is restricted to 16 , as is the number of stations that may be active at one time.

In the current version of NOVA SKED, the only mass data collection device allowable is magnetic tape. Data is written to mag tape in RDOS-compatible binary format. To avoid loss of data, there are two doublebuffer systems, one for each individual station and one for the mag tape. The buffer size for a station is equal to the number of recording counters specified by the SKED program.

Since the mag tape is not a file-structured device, data from all stations running simultaneously must go in the same file. Further, the data is dumped randomly as the DUMP command is issued for each particular station. In order that data be identifiable, the data from each station for each mag-tape dump is preceded by a block of "headers," which identify the station number and other relevant bookkeeping information such as the subject number, experiment number, group number, and date. Also included is a program number for a permanent record of the program loaded into that station, which is especially handy when parameters of a program are being changed routinely. The first of these headers is the number of recording counters associated with that station, so that data may be easily sorted using that number. 
When the last station active at a particular time ends, the mag tape file is automatically closed and a double end of file (EOF) written on the tape. The tape is then automatically positioned between the two EOFs in preparation for further data collection. The tape can be positioned from the keyboard by either specifying the file number or searching for the double EOF.

\section{ADDITIONAL RTS COMMANDS}

There are several important SKED monitor commands that have not been discussed in previous sections, including the following: (1) The mag-tape file may be closed from the keyboard, and data may be printed to either the Teletype or the mag tape manually. (2) A station may be stopped from the keyboard, with all $\mathrm{I} / \mathrm{O}$ shut off; the mag-tape buffer is dumped and the file closed if that is the last station running. (3) An active station may be removed from the RTS scan with no change in $\mathrm{I} / \mathrm{O}$, and restarted in the condition in which it was stopped. This is particularly useful for testing and debugging purposes. (4) Another useful feature for testing the hardware (or rinsing out feeders) is a command that allows turning any bit on or off; this command may be issued while stations are active but is independent of the SKED relocatable binary programs. (5) Responses on input lines may also be simulated from the keyboard, but this can be done only when a program is loaded into a particular station. This is typically the way in which an experimental procedure is activated in a station, and may also be used for hardware or software debugging. (6) Any variable in a SKED program may be examined or modified from the keyboard while the program is running. This is useful during training as well as in schedules where it is desirable to start "in a different place" each session (e.g., the variable interval schedule).

\section{DATA SORTING AND ARCHIVAL STORAGE}

Although data untangling and storing is not strictly part of the SKED system, it may be useful to describe a general strategy that has been developed using a set of FORTRAN programs run under RDOS. The first step is to sort the SKED-generated files by subject number and write the data into RDOS disk files. This can be done on any number of files automatically until the double EOF is encountered or by specified file numbers. Each disk file name is extracted from the header information and consists of the experiment number and date in month, day, year with the subject number as the extension. This is preceded by the letter D for easy RDOS manipulation; for example, D $\$ 3 \emptyset 1 \emptyset 177.78$ refers to Experiment 3, January 1, 1977, Subject 78 . The disk file consists of the headers followed by the session data in a continuous list, with all header dumps after the first one eliminated. This SORT program also creates a file containing all file names generated on a particular run of the program. The analysis program for individual experiments may take its input file list from the file of file names created by the sorting program, using the experiment number as a mask for relevant files.

The most convenient data form for archival storage is that generated by the sorting program, that is, one subject's data for one session per file with the identifying file name. Data is stored on mag tape in the RDOS DUMP format, so that one mag-tape file may contain any number of disk files. Individual tapes are used for each experiment number, with the first magtape file being an ASCII file containing the tape number, experiment number, and date of creation. A set of FORTRAN programs keeps track of the current tape number, file number for dumping, and so on, for each experiment to insure that data is not dumped onto the wrong tape or into the wrong file. Two duplicate copies of all data are generated independently.

\section{COMING ATTRACTIONS}

A disk-based SKED system that runs under RDOS control is currently in use at Health and Welfare Canada. This system is compatible with a foreground-background system, so that SKED need occupy only part of the machine and other functions not requiring real-time priority, such as data analysis and editing, could coexist with the running of SKED programs.

For more detailed information on NOVA SKED, contact the authors at Health and Welfare Canada, Tunney's Pasture, Ottawa, Ontario, Canada K1A OL2.

\section{REFERENCE NOTE}

1. Snapper, A. G., Stephens, K. R., Cobez, R. I., \& Van Haaren, F. The SKED software system-Manual 2: $O S / 8$ and time share SKED. Kalamazoo, Mich: The SKED Users Group, March 1976.

\section{REFERENCES}

SNAPper, A. G. Use of a notational system for digital control and recording. Behavior Research Methods \& Instrumentation, 1973, 5, 124-128.

Snapper, A. G., \& Kadden, R. M. Time-sharing in a small computer through the use of a behavioral notation system. In B. Weiss (Ed.), Digital computers in the behavior laboratory. New York: Appleton-Century-Crofts, 1973. Pp. 41-97.

SNAPPER, A. G., KNAPP, J. Z., \& Kushner, H. K. Mathematical description of schedules of reinforcement. In W. N. Schoenfeld (Ed.), The theory of reinforcement schedules. New York: Appleton-Century-Crofts, 1970. Pp. 247-275.

\section{NOTES}

1. NOVA is a registered trademark of Data General Corporation, Southboro, Massachusetts. SKED is a registered trademark of State System, Inc., for its brand of computer interface, software, and service associated therewith.

2. Available from Computer Products, Inc., Fort Lauderdale, Florida.

(Received for publication January 24, 1978; revision accepted April 20, 1978.) 